\title{
Cognitive Map: Universal Code for Learning and Memory
}

\author{
Ran Cheng \\ ${ }^{1}$ School of Pharmaceutical Sciences, Xiamen University, Xiamen City, Fujian Province, 361102, China \\ *Corresponding author. Email:rcheng1004@stu.xmu.edu.cn
}

\begin{abstract}
Ever since the discovery of place cells, the conception of the cognitive map has revived and the intriguing assumption that spatial navigation system also encodes other cognitive functions has aroused interest among neuroscientists. Though the hypothesis has been viewed with some skepticism, evidence gradually accumulated in the last decade, especially with regard to more abstract functions of the human brain. Drawing on the most recent experimental results, this theoretical literature review attempted to further assess the possibility that the human brain processes various kinds of information by constructing cognitive maps and uncover some of its governing principles. Conclusions are that recent evidence generally supports the hypothesis, though the characteristics of spatial map and cognitive map exhibit differences. Moreover, time scale and reference frame are two variables that determine the neural activity during cognitive tasks, more specifically, the brain regions that exhibit signals that are thought to reflect the participation of cognitive maps vary with changes in time scale and reference frame. Therefore, these two variables should be taken into account when designing experiments and interpreting the results. The review will help guide future exploration of the cognitive map, which is a promising candidate for unraveling the universal rule of human cognition.
\end{abstract}

Keywords : Cognitive map, Spatial navigation, Hippocampus, Medial temporal lobe, Prefrontal cortex

\section{INTRODUCTION}

One of the mysteries of the human brain is that how it generates concepts, develops structure from novel information and makes decisions based on previous experience, which are essential for adaptive behavior[1]. Recent progress suggests that the medial temporal lobe (MTL) might underline higher-level cognitive functions other than spatial navigation. According to this proposition, place cells in the hippocampus, grid cells in the entorhinal cortex, as well as other types of cells related to spatial navigation may support the construction of a cognitive map for a broad range of cognitive functions governed by geometric principles[2]. Assessing the validity of this possibility and identifying its material basis will by no means benefit human intelligence research and artificial intelligence.

However, research into the hypothesis started relatively late and evidence is limited, partly due to the transition from animals to humans and the complexity of the experimental design. A review article by Bellmund et al. explicitly discussed the theoretical possibility and the experimental evidence of the idea up to that point[2]. This theoretical paper will summarise the exploration of cognitive maps after that review and discuss to what extent their ideas are developed, supported or rejected by the most recent evidence. Only the experiments that targeted humans are included in this review, regardless of the methodology.

The main body of this review is divided into two parts. In the first part, the relationship between spatial map and cognitive map will be discussed. Then in the second part, time scale and reference frame, two variables that have not been pointed out by Bellmund et al. and are neglected by some researchers, will be highlighted, in hope of offering better explanations of research results, aiding further clarification of the concept and guiding future research into its neural mechanisms. 


\section{RELATIONSHIP BETWEEN SPATIAL MAP AND COGNITIVE MAP}

\subsection{Spatial Navigation System Supports Cognitive Maps}

Recent studies provide further evidence to the view that the spatial navigation system also underlines higher cognitive functions[2], although investigations into humans could only employ non-invasive methods like functional magnetic resonance imaging (fMRI), and could not directly observe the activity of place cells or grid cells. Two codes that are believed to be associated with the activity of these cells in the blood-oxygenation-level-dependent (BOLD) signal detected by fMRI are the grid-like code and distance code, which serve as an indirect and preliminary measurement of the activation of the spatial navigation system[2][3].

One recent study discovered that the neural activity in the entorhinal cortex and the medial prefrontal cortex, which is required for spatial navigation, encoded the Euclidean distance over a two-dimensional space between entities, whereas the hippocampus reinstates the Euclidean distance between two entities for memory retrieval[4]. This indicates that abstract information about people's social hierarchy is automatically combined to construct a low dimensional map in the human brain, allowing for novel inference based on former experience.

Conceptual knowledge is one significant development in this regard[5], though direct experimental evidence is scarce. Computational modeling suggests that one successful model of human concept learning, the clustering learning model, can be safely applied to spatial contexts, indicating a common learning neural mechanisms for space and concepts[6]. Moreover, drawing lessons from the hippocampal-entorhinal system, a computational model, the Visual Concept Space Model (VCSM), has been proposed to account for human visual concept learning and achieved better performance compared to baseline methods[7]. However, computational results only provide theoretical support of the proposal that concepts are organized into cognitive maps, but cannot serve as concrete evidence.

Another high-level cognitive ability that the cognitive map hypothesis has extended to is semantic knowledge. Simone Vigano et al. conducted two experiments investigating the processing of semantic words in the human brain and found that after training, the medial prefrontal cortex and the right entorhinal cortex encoded the distance between concepts, whereas the latter also exhibited a periodic modulation, which the researchers suggest serve as a function of traveled direction[3][8], though the possibility that perceptual coding also played a rule cannot be fully ruled out. One important implication of their work is that they employed multisensory stimulus and discovered the distance-based code in visual areas and audio areas respectively, suggesting that the meaning of words, at least when they can be organized long two feature dimensions, is represented in the human brain across multiple maps of different dimensionality[3].

\subsection{Differences Between Spatial Map and Cognitive Map}

The characteristics of spatial and non-spatial map exhibit differences[6][9]. Employing a within-subject design and computational modeling, one group of researchers found that although the same Gaussian Process model best captured human search decisions and judgements in both spatially and conceptually correlated reward environments, the level of uncertainty-directed exploration is lower in the conceptual domain and the level of random exploration vice versa[9].

Furthermore, it is theoretically unknown whether spatial cognition is merely one function of a more universal cognitive system[6], or the system is primarily constructed for spatial navigation but is recruited for higher cognitive functions in humans[2][8]. Although the two different views have been put forward in some studies as the implications of the results, no research can distinguish these two possibilities. From an evolutional point of view, a simple fact is that animals and humans deal with spaces or images from the start, whereas the ability to think abstractly and construct concepts is a much later development. Therefore, this review holds the view that the spatial navigation system inside the brain evolved to allow representation of other stimuli such as concepts or semantic knowledge, at least when the concept can be defined by values along the feature dimensions.

\section{TWO VARIABLES: TIME SCALE AND REFERENCE FRAME}

\subsection{Cognitive Maps Across Different Time Scales}

At least two brain regions that are sensitive to time scale are the hippocampus and the prefrontal cortex. There has been a debate regarding the role of the hippocampus in short-term or long-term memory for some time, the reason why the research by Vigano and Piazza did not found significant changes in the BOLD signals in the hippocampus is possible that the knowledge had already become consolidated into cortex over the nine days of training, and therefore was no longer hippocampally dependent[10]. One study attempting to reconcile the role of hippocampus in 
short-term and long-term memory states that hippocampus contributes to long-term memory through remarkably short-term processing[11]. They employed a scene viewing task and identified hippocampal contributions to guide forthcoming fixations, eye movements correlating with theta oscillations, which are thought to reflect place cell replay[2].

The prefrontal cortex has long been associated with attention and working memory, a form of short-term memory[12][13]. Cognitive map is a flexible low-dimensional system in which variables that are relevant are represented by cell assemblies and stimuli or concepts are mapped into the cognitive space according to their values along the feature dimensions[2][6][14]. In experimental paradigms that requires working memory, the first step in constructing the cognitive map is to define the dimensions, whether consciousness is aware of this process. Whatever the factors that the cells are sensitive to, such as sound, odor, time, converging evidence suggests that the dimensions are decided by task demands and modulated by medial prefrontal cortex[6][15]. In this case, instead of recruiting other sets of cells, the same populations of place cells that are activated remap when task demand changes[2]. This coincides with one constraint on human cognition - the inability to think of two goals at a one time-point.

\subsection{Cognitive Maps Constructed from Different Reference Frames}

Recent work suggests a dissociation between spatial maps that are self-centred and world-centred, each with different neural mechanisms. The self-centred mental map is often referred to as cognitive map represented by the hippocampal-entorhinal system, whereas the world-centred map is termed image spaces represented by the parietal cortex[16][17].

Nevertheless, it can be difficult to detangle in some experimental paradigms and some studies employed the term cognitive map when mental navigation actually relies on both mechanisms. One example of this is the study that extends the idea that multiple scales of coding might be reflected by the gradient along the hippocampal axis by comparing the BOLD signal in the human brain when the subjects undertake spatial navigation tasks across different spatial scales[2]. Naturally, as the spatial scale expands from room to continent, the mental map is more allocentric than egocentric. Despite identifying three gradients that varied with coding scale in parahippocampal place area, retrosplenial complex and occipital place area respectively, they also found several other regions, including the parietal cortex, that displayed the highest activity in the smallest navigating space and decreased activity with increasing scale[18]. These results coincide with the assumption that image spaces are represented in the parietal cortex and suggest that there is an overlap between the brain regions involved in cognitive map and image spaces.

Another study targeted at social cognition investigated how the human brain process social connections and interactions and found that generally consistent with other studies that investigated social information in humans[4][18], the social network distance information is represented in the default mode network[17]. However, the study discovered a cortical division between the neural representations of social network structure, self-referenced social distance, and trait-based social knowledge[17].

The distinction between the neural activity of egocentric and allocentric mental maps might reflect two distinct processes in human learning. Studies have shown that cognitive maps also play a role in adaptive learning, whose neural mechanisms can be divided into two processes, neural mechanisms of surprise promoting state transition and neural representation of states and transition matrices[1]. Presumably, the expectation of state and the expectation of reward correspond with egocentric and allocentric mental maps respectively. It is possible that both maps contribute to expectation updating, with which learning occurs. However, the link between cognitive map and learning is yet to be established and the exact mechanisms of how different systems interact remain elusive.

\section{CONCLUSIONS}

Converging evidence in the past few years suggests that cognitive maps serve as a representation format of various forms of information, allowing for higher-level cognition in humans, such as episodic memory and working memory in adaptive learning. However, cognitive maps and spatial maps have different properties, which are yet to be clarified. Possibilities are that cognitive maps are not specific to the hippocampal-entorhinal system or the cells that are supposed to support spatial navigation, as indicated by Bellmund et al. in their review in 2018, but are present in some other brain regions as well, indicating a more general mechanism of the human mind to process information.

Current literature provides mixed results concerning the regions in the human brain that exhibit grid-like code or distance code and the interpretation of the functions of these areas are diverse. Future research should take into account time course and reference frames as two variables when designing the paradigm or discussing the results, so as to further clarify the mechanisms of cognitive maps and the functions of different brain regions. Furthermore, it is worth noting that the distinction between egocentric and allocentric maps might also be reflected in learning. 
Computational models in human learning spatial navigation should help identify the processing steps in cognitive tasks, based on which experimental paradigms can be designed to investigate the neural mechanisms underlining high-level cognition in humans. Moreover, the exact role of cells in the spatial navigation system, such as place cells and grid cells in the medial temporal lobe is still under debate and resolving this question relies on animal studies or more advanced techniques measuring the human brain. Intriguing questions remain that what cellular or molecular structure of the nervous system enables this form of representation and why the human brains are constructed this way.

\section{ACKNOWLEDGMENTS}

I would like to offer my sincere thanks to the people below, without whose help this review would not have been completed. First of all, I was inspired to write on this topic by the online course delivered by Prof. Kate J. Jeffery-The Behavioural Neuroscience of Learning, Memory and Navigation. Next, I am grateful for the teaching assistant of the course, Gongting Wang, who discussed relevant questions and ideas with me and gave me a lot of inspirations. Finally, the dissertation adviser, Yufan Huang, provided me with guidance on the structure and the grammar of the dissertation. Furthermore, my three classmates, Yingcai Hu, Yunze $\mathrm{Gu}$ and Yishu Sun, who come from different scientific backgrounds and were always active and enthusiastic during class, also stimulated me to a considerable extent.

\section{REFERENCES}

[1]Yu, L. Q., Wilson, R. C., \& Nassar, M. R. (2021). Adaptive learning is structure learning in time. Neuroscience and Biobehavioral Reviews, 128, 270-281. doi:10.1016/j.neubiorev.2021.06.024.

[2]Bellmund, J. L. S., Gardenfors, P., Moser, E. I., \& Doeller, C. F. (2018). Navigating cognition: Spatial codes for human thinking. Science, 362(6415), 11. doi:10.1126/science.aat6766.

[3]Vigano, S., Rubino, V., Di Soccio, A., Buiatti, M., \& Piazza, M. (2021). Grid-like and distance codes for representing word meaning in the human brain. Neuroimage, 232. doi:10.1016/j.neuroimage.2021.117876.

[4]Park, S. A., Miller, D. S., Nili, H., Ranganath, C., \& Boorman, E. D. (2020). Map Making: Constructing, Combining, and Inferring on Abstract Cognitive Maps. Neuron, 107(6), 1226-+. doi:10.1016/j.neuron.2020.06.030.

[5]Morton, N. W., \& Preston, A. R. (2021). Concept formation as a computational cognitive process.
Current Opinion in Behavioral Sciences, 38, 83-89. doi:10.1016/j.cobeha.2020.12.005.

[6]Mok, R. M., \& Love, B. C. (2019). A non-spatial account of place and grid cells based on clustering models of concept learning. Nature Communications, 10. doi:10.1038/s41467-019-13760-8.

[7]Sheng, H., Mo, H., \& Moanda Ndeko Mosengo, C.-M. (2021). A Hippocampal-Entorhinal System Inspired Model for Visual Concept Representation. Ieee Transactions on Cognitive and Developmental Systems, 13(2), 429-441. doi:10.1109/tcds.2020.2978918

[8]Vigano, S., \& Piazza, M. (2020). Distance and Direction Codes Underlie Navigation of a Novel Semantic Space in the Human Brain. Journal of Neuroscience, $\quad 40(13), \quad 2727-2736$. doi:10.1523/jneurosci.1849-19.2020.

[9]Wu, C. M., Schulz, E., Garvert, M. M., Meder, B., \& Schuck, N. W. (2020). Similarities and differences in spatial and non-spatial cognitive maps. Plos Computational Biology, 16(9). doi:10.1371/journal.pcbi.1008149.

[10]Bokeria, L., Henson, R. N., \& Mok, R. M. (2021). Map-Like Representations of an Abstract Conceptual Space in the Human Brain. Frontiers in Human Neuroscience, 15. doi:10.3389/fnhum.2021.620056.

[11]Kragel, J. E., Schuele, S., VanHaerents, S., Rosenow, J. M., \& Voss, J. L. (2021). Rapid coordination of effective learning by the human hippocampus. Science Advances, 7(25). doi:10.1126/sciadv.abf7144.

[12]Christophel, T. B., Klink, P. C., Spitzer, B., Roelfsema, P. R., \& Haynes, J. D. (2017). The Distributed Nature of Working Memory. Trends in Cognitive Sciences, 21(2), 111-124. doi:10.1016/j.tics.2016.12.007.

[13]Constantinidis, C., \& Klingberg, T. (2016). The neuroscience of working memory capacity and training. Nature Reviews Neuroscience, 17(7), 438-449. doi:10.1038/nrn.2016.43.

[14]Nieh, E. H., Schottdorf, M., Freeman, N. W., Low, R. J., Lewallen, S., Koay, S. A., . . Tank, D. W. (2021). Geometry of abstract learned knowledge in the hippocampus. Nature, 595(7865), 80-+. doi:10.1038/s41586-021-03652-7.

[15]Mack, M. L., Preston, A. R., \& Love, B. C. (2020). Ventromedial prefrontal cortex compression during concept learning. Nature Communications, 11(1), 11. doi:10.1038/s41467-019-13930-8. 
[16]Bottini, R., \& Doeller, C. F. (2020). Knowledge Across Reference Frames: Cognitive Maps and Image Spaces. Trends in Cognitive Sciences, 24(8), 606-619. doi:10.1016/j.tics.2020.05.008.

[17]Peer, M., Hayman, M., Tamir, B., \& Arzy, S. (2021). Brain Coding of Social Network Structure. Journal of Neuroscience, 41(22), 4897-4909. doi:10.1523/jneurosci.2641-20.2021.

[18]Peer, M., Ron, Y., Monsa, R., \& Arzy, S. (2019). Processing of different spatial scales in the human brain. Elife, 8. doi:10.7554/eLife.47492. 